\title{
Influence of twin boundary orientation on magnetoresistivity effect in free standing $3 \mathrm{C}-$ $\mathrm{SiC}$
}

\author{
Remigijus Vasiliauskas, A. Mekys, P. Malinovskis, Mikael Syväjärvi, \\ J. Storasta and Risitza Yakimova
}

\section{Linköping University Post Print}

N.B.: When citing this work, cite the original article.

Original Publication:

Remigijus Vasiliauskas, A. Mekys, P. Malinovskis, Mikael Syväjärvi, J. Storasta and Risitza Yakimova, Influence of twin boundary orientation on magnetoresistivity effect in free standing 3C-SiC, 2012, Materials letters (General ed.), (74), 203-205.

http://dx.doi.org/10.1016/j.matlet.2012.01.120

Copyright: Elsevier

http://www.elsevier.com/

Postprint available at: Linköping University Electronic Press http://urn.kb.se/resolve?urn=urn:nbn:se:liu:diva-76368 


\title{
Influence of Twin Boundary Orientation on
}

\section{Magnetoresistivity Effect in Free Standing 3C-SiC}

\author{
R. Vasiliauskas ${ }^{\mathrm{a} *}$, A. Mekys ${ }^{\mathrm{b}}$, P. Malinovskis ${ }^{\mathrm{b}}$, M. Syväjärvi ${ }^{\mathrm{a}}, \mathrm{J}$ Storasta $^{\mathrm{b}}$ \\ and R. Yakimova ${ }^{\mathrm{a}}$
}

${ }^{a}$ Department of Physics, Chemistry and Biology, Linköping University, SE-581 83, Linköping, Sweden.

${ }^{\mathrm{b}}$ Institute of Applied Research, Vilnius University, LT 10222, Vilnius, Lithuania.

*Corresponding Author : Tel: +46-13-281704. Fax: +46-13-137568. E-mail: remis@ifm.liu.se.

\begin{abstract}
Free standing 3C-SiC (111) samples with differently oriented twin boundaries were prepared using on-axis and slightly off-axis $6 \mathrm{H}-\mathrm{SiC}$ substrates. The orientation of twin boundaries causes either an enhancement or suppression of the magnetoresistance mobility. The origin of carriers mobility difference is attributed to the specific structure of these defects. The height of the barriers created by twin boundaries was found to be $0.2 \mathrm{eV}$
\end{abstract}

Keywords. Magnetoresistance, carrier mobility, twin boundaries, 3C-SiC.

\section{Introduction}

Among the large variety of $\mathrm{SiC}$ polymorphs only the $3 \mathrm{C}-\mathrm{SiC}$ polytype has a cubic lattice and its physical properties are isotropic. In addition it has been indicated that the 
cubic SiC maybe a good substrate for growth of graphene [1]. The smaller band gap $(2.23 \mathrm{eV})$ compared to hexagonal polytypes lower the interface state density in the 3C$\mathrm{SiC} / \mathrm{SiO}_{2}$ and 3C-SiC/graphene systems, thus ensuring a higher channel mobility in the respective electronic devices. It has been theoretically predicted that $30 \%$ efficiency of solar cells produced from 3C-SiC can be achieved [2].

The formation of twin boundaries (TBs) is one of the main problems in the growth of 3C-SiC on $6 \mathrm{H}-\mathrm{SiC}$ substrates. Material with large density of extended defects cannot be assessed precisely by Hall effect measurement. Instead, the magnetoresistance (MR) effect can be utilized to acquire e.g. carrier mobility. However, only few publications are available on the TBs influence on MR measurements $[3,4]$ and not much is known about the electrical properties of TBs. Additionally, it is known that defects having specific distribution can induce anisotropy in carrier mobility which is of importance for the device design $[5,6]$. In this work we have prepared free standing $3 \mathrm{C}$ $\mathrm{SiC}$ with differently orientated TBs and show that their presence and direction can induce anisotropy in carrier mobility extracted from the magnetoresistivity effect.

\section{Experimental}

Growth of bulk-like 3C-SiC (111) was performed by sublimation epitaxy [7]. Two samples were grown on large area (quarters of two inch) (0001) silicon face 6H-SiC substrates. The first sample was grown on nominally on-axis $\left(<0.5^{\circ}\right.$ off-axis $)$ substrate and the second one was grown on slightly off-axis $\left(\sim 1^{\circ}\right)$ substrate. A growth temperature of $1775^{\circ} \mathrm{C}$ and temperature ramp up of $5 \mathrm{~K} / \mathrm{min}$ was applied for all samples. At these conditions growth rate was $\sim 260 \mu \mathrm{m} / \mathrm{h}$. After growth the $6 \mathrm{H}-\mathrm{SiC}$ substrate was removed and the surface roughness was minimized by mechanical polishing to have free standing 
$3 \mathrm{C}$-SiC material with thickness of $\sim 250 \mu \mathrm{m}$. The unintentional doping of the material was $\mathrm{N}_{\mathrm{D}} \approx 4.4 \times 10^{16} \mathrm{~cm}^{-3}$ and $\mathrm{N}_{\mathrm{A}} \approx 4.0 \times 10^{14} \mathrm{~cm}^{-3}$.

Seven 3C-SiC samples were prepared in Hall-bar geometry by cutting into pieces of $2.0 \mathrm{~mm}$ width and $5.0 \mathrm{~mm}$ length. Samples from the first wafer were cut in the way that TBs have random orientation (Fig. 1a,b). Samples from the second wafer were cut with TBs parallel (Fig. 1c,d) and perpendicular (Fig. 1e,f) to the long edge of the sample and also the current flow. For comparison, one sample was cut from the second wafer region with a very low TB density (Fig. 1g). To have more reliable data two samples of each TB orientation were prepared, except the one with low density of TBs. Ni contacts were deposited and additionally covered with Au using physical vapor deposition (PVD) technique through a $\mathrm{Cu}$ mask at $400^{\circ} \mathrm{C}$ in a vacuum chamber and annealed at $1100^{\circ} \mathrm{C}$ for 10 minutes in air. All electrical measurements were performed in dark.

We have previously shown [8], that the $3 \mathrm{C}-\mathrm{SiC}$ nucleation on on-axis $6 \mathrm{H}-\mathrm{SiC}$ substrates is preceded by homoepitaxial $6 \mathrm{H}-\mathrm{SiC}$ growth in a spiral mode. Cubic $\mathrm{SiC}$ starts to form by 2D-nucleation mechanism when the $6 \mathrm{H}-\mathrm{SiC}$ spiral terraces are large enough. The 3C-SiC nuclei appear nearly uniformly distributed all over the sample. In the next phase these nuclei expand in all directions (normal and lateral) until they meet other nuclei and merge, or TBs are formed between them if they are twinned. After that they expand only in the normal (111) growth direction. Thus in case of using on-axis substrates a random pattern of TBs is obtained (Fig. 1a,b).

A different situation appears when growth of cubic SiC takes place on slightly off-oriented $\left(\sim 1^{\circ}\right) 6 \mathrm{H}-\mathrm{SiC}$ substrates. Similarly to on-axis substrates the growth commences by homoepitaxial $6 \mathrm{H}-\mathrm{SiC}$. However, the latter is formed in step flow mode instead of spiral growth. In this case the nucleation of 3C-SiC does not begin over the 

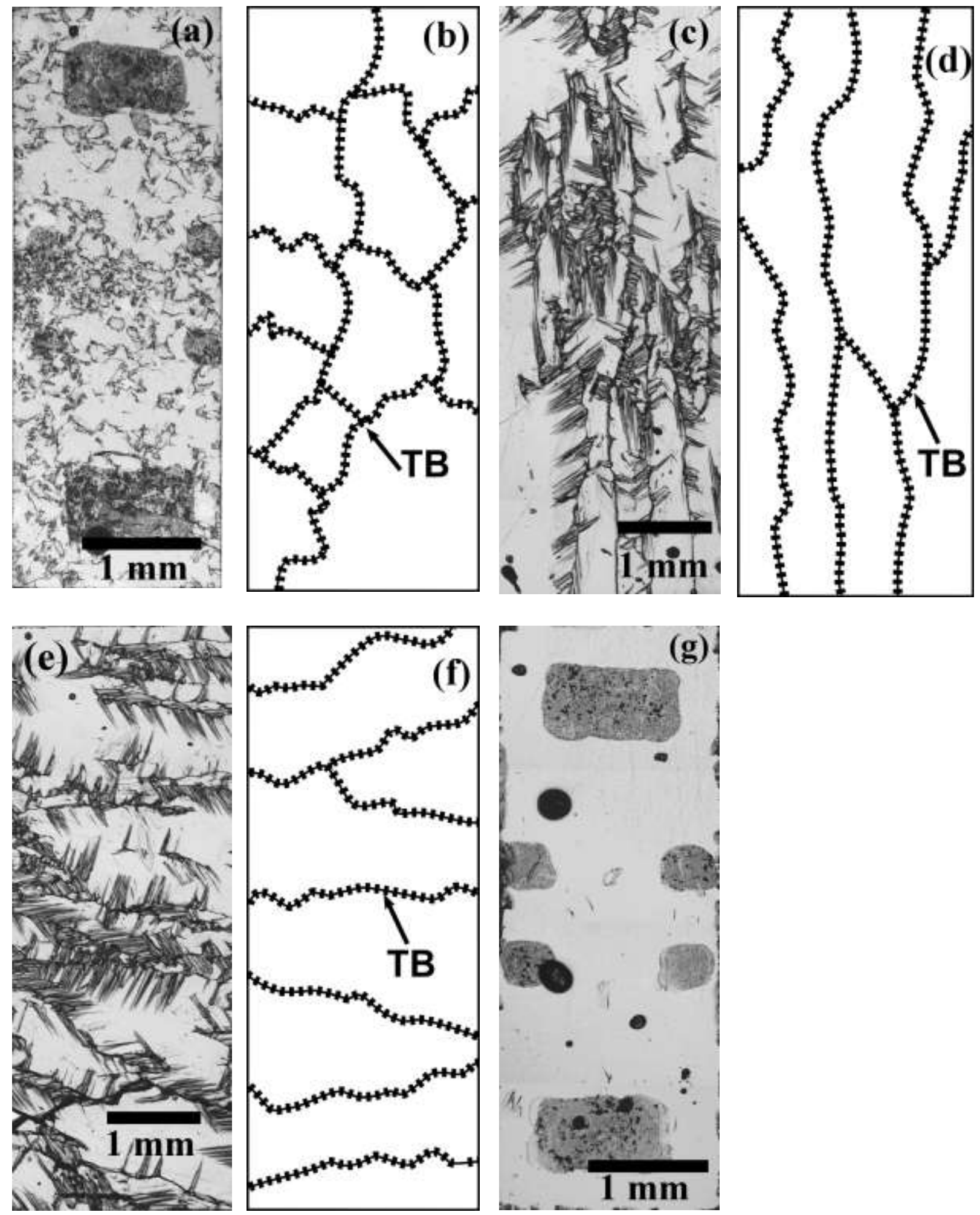

Fig 1. Optical micrographs and sketch of samples prepared for electrical measurements with different TBs orientation: $a, b)$ random; c, d) parallel; e, f) perpendicular; g) with low density of TBs ((a) and (g) samples are with contact areas). 
whole substrate, but on the basal plane which has formed at the edge of the sample from where steps start and flow along off-axis direction. In other places the step terraces are too small and the supersaturation is not high enough for 3C-SiC to nucleate [8]. Initially the $3 \mathrm{C}-\mathrm{SiC}$ nuclei on the basal plane expand in random directions until they meet other nuclei, similarly to growth on on-axis substrates. In the course of growth the basal plane area expands in the off-cut direction of the sample. This drives the $3 \mathrm{C}-\mathrm{SiC}$ domains to laterally grow in the same direction as the step flow growth $[9,10]$. In this way, the 3CSiC enlarge and cause directional domains (Fig. 1c-f).

The cubic SiC polytype was identified by the color (yellow for $3 \mathrm{C}$ and transparent/blue for $6 \mathrm{H})$ in an optical microscope $(\mathrm{OM})$ in transmission light and confirmed using Raman spectroscopy. The surface morphology of the grown layers was studied with OM. The magnetoresistance measurements were performed in a Hall mobility setup [11]. A magnetic field strength of $1.7 \mathrm{~T}$ was applied in the experiments. A Keithley 6430 was used as the voltage source (10V was applied) and the electric current measurement unit. The measurements were performed in the vacuum cryostat (base vacuum of $10^{-3} \mathrm{mbar}$ ) in temperature range from $100 \mathrm{~K}$ to $400 \mathrm{~K}$.

\section{Results and discussions}

From the MR measurements carrier mobilities were derived [11] at different temperatures (Fig. 2a). There is a noticeable difference in the impact of the parallel and perpendicular TBs on the MR mobility. This is especially prominent at low temperatures. In the crystals with the TBs orientation along the electric current flow, the MR mobility is 4.5 times greater (at low temperatures) than in the samples where TBs orientation is perpendicular to the current flow direction. The MR mobility in samples with random TB orientation is close to that of the samples with perpendicular TB $(\sim 250$ - 
$300 \mathrm{~cm}^{2} / \mathrm{Vs}$ ) and almost independent from temperature. This may be expected because some of the TBs have also perpendicular orientation which lowers the carrier mobility. In the sample with a low density of TBs the MR mobility is very close to the average mobility of samples with parallel and perpendicular TBs.

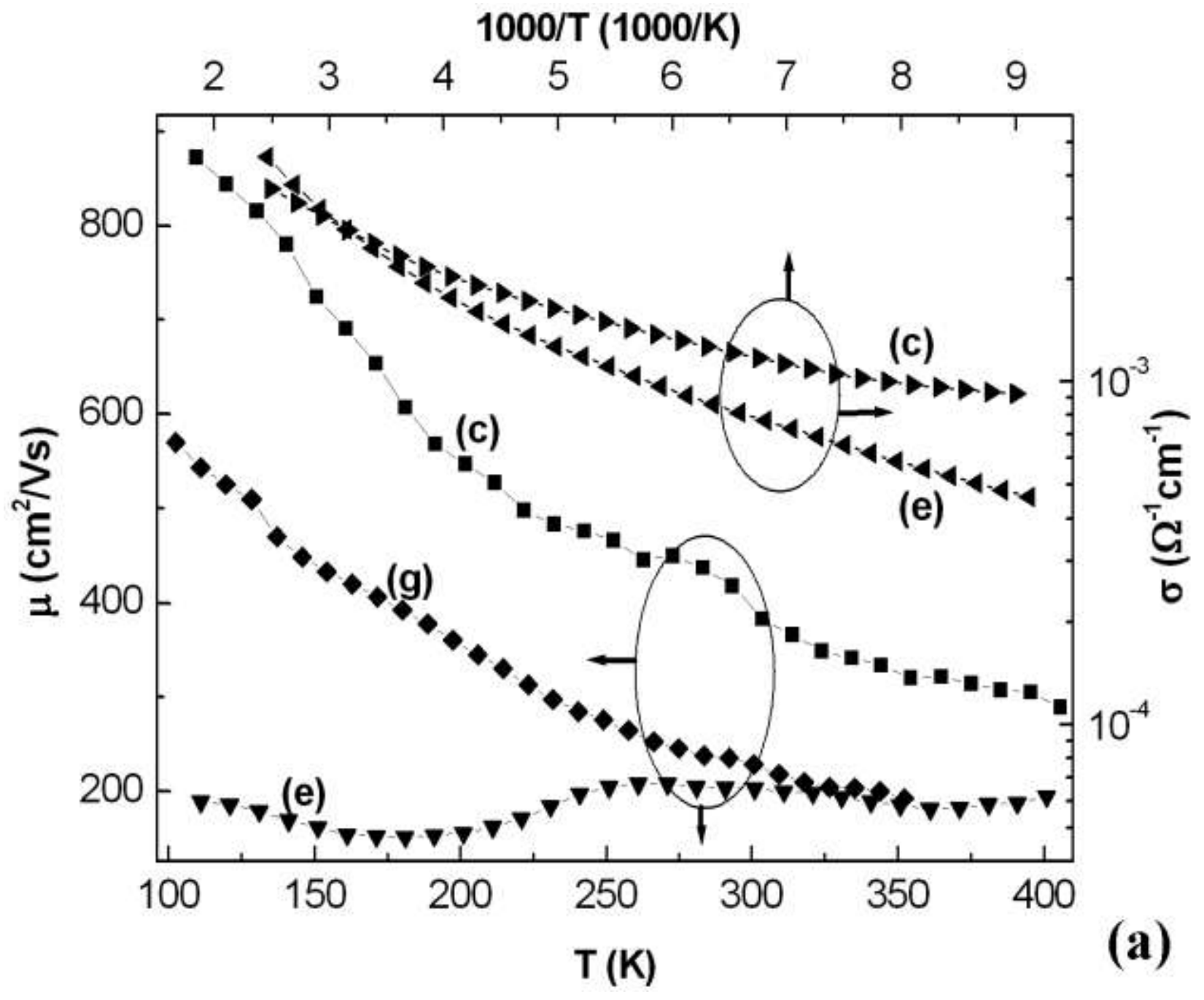




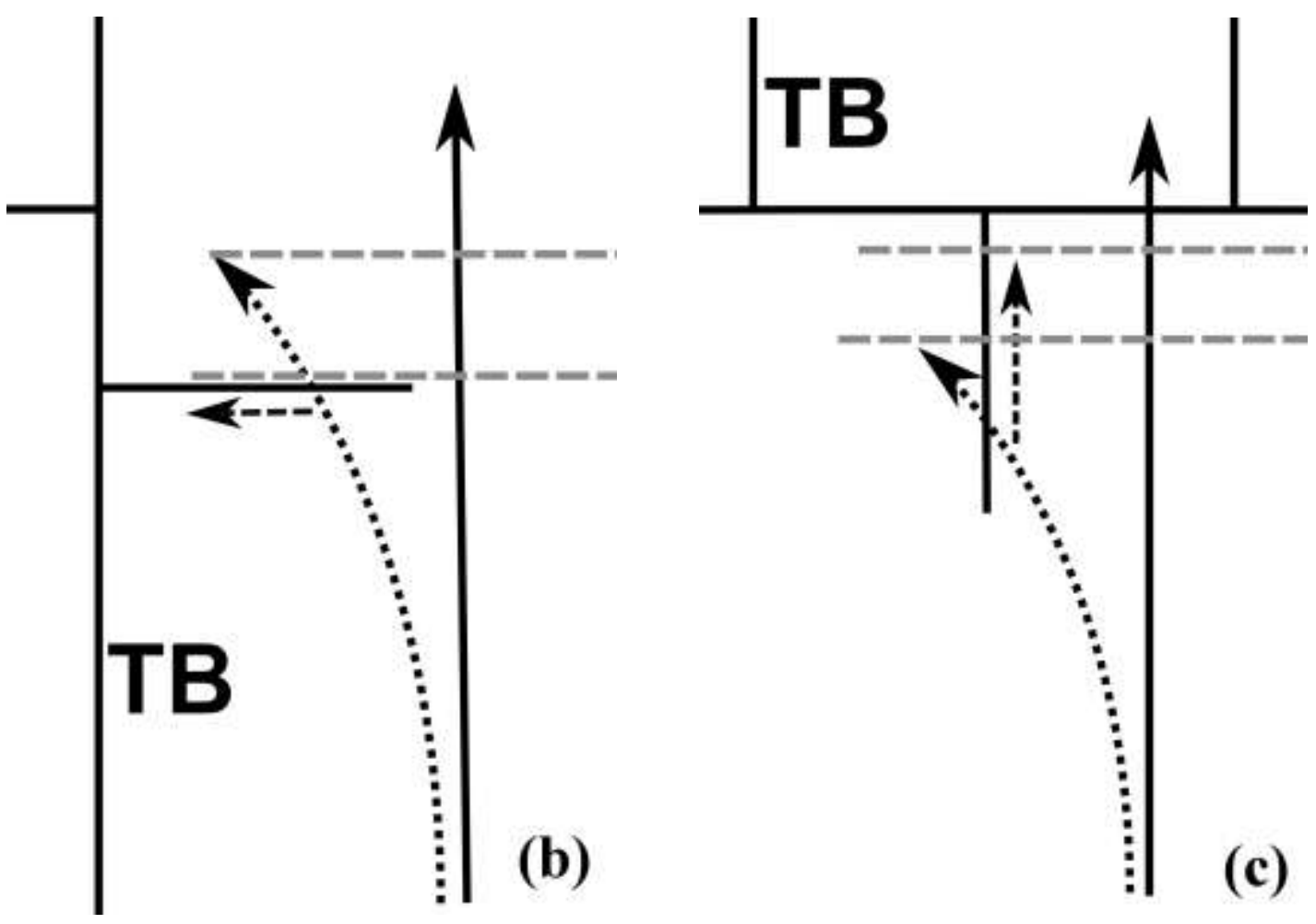

Fig 2a. MR mobility vs. temperature for samples: c - parallel; e - perpendicular; $\mathrm{g}$ - low TB density and conductivity vs. reciprocal temperature (upper curves). (b) MR effect enhancement when TBs are parallel; (c) suppression when TBs are perpendicular. A solid arrow represents path of electron without a magnetic field, dotted arrow - e path in a magnetic field, when TB are not present, dashed arrows - e path in a magnetic field when TBs are present, dashed lines - difference in path length with and without TBs.

It is commonly accepted that in the samples with higher density of defects the carrier mobility should be lower compared to the ones with lower defect density. It was shown that in 3C-SiC a higher density of TBs lowers the MR mobility [4]. In our case, samples with perpendicular TBs are in good agreement with this observation (Fig. 2a). In these the carrier mobility is lower $\left(200 \mathrm{~cm}^{2} / \mathrm{Vs}\right.$ at $\left.110 \mathrm{~K}\right)$ compared to the samples with low density of TBs $\left(580 \mathrm{~cm}^{2} / \mathrm{Vs}\right.$ at $\left.110 \mathrm{~K}\right)$. This is especially noticeable at low temperatures. However, samples with parallel TBs show completely opposite results and the MR mobility increases $\left(880 \mathrm{~cm}^{2} / \mathrm{Vs}\right.$ at $\left.110 \mathrm{~K}\right)$ compared to the samples with low density of TBs. It is, however, not in a correlation with the conductivity behavior (Fig. 2a). It appears that the mobility change is not related with the doping difference (if there 
is any), neither with defect scattering. Thus we believe that the observed phenomenon in these experiments is either due to an enhancement or suppression of the MR effect by TBs.

The enhancement or suppression of MR can be explained by considering the structure of TBs in Fig. 1. The single TB is always decorated with branches like a fir tree which also are TBs. For example, in samples with parallel TBs the carriers in a magnetic field are bent from the path along the sample to the branches and there they can be trapped or more intensively scattered (Fig. 2b). By this, the MR increases. While in the samples with perpendicular TBs, the branches capture the carriers which are trying to bend away from the direct path in the magnetic field and direct them in a straight way (Fig. 2c). Thus in the magnetic field carriers' path is changed less and the MR increases less.

At higher temperatures the MR for all samples becomes similar, indicating that the effect of TB orientation is significantly lower. This is caused by the increased carrier density. The relative contribution from carriers passing the TB rich regions is being diminished as more carriers contribute to the total electrical current. Additionally, thermal activation energy of $0.20 \mathrm{eV}$ was found from the carrier concentration dependence on temperature. We believe this activation energy has originated from the TBs which act as barriers and carriers have enough energy to overcome them only at higher temperatures.

\section{Conclusions}

We have prepared bulk-like 3C-SiC (111) samples with differently oriented twin boundaries. The orientation of TBs was obtained by growth using on-axis and slightly off-axis substrates. Compared to the samples with few TBs, in samples with larger 
density of TBs the MR mobilities are enhanced where TBs are parallel and diminished where TBs are perpendicular to the current flow. This is less pronounced at higher temperature as relatively less carriers have to overcome barriers $(0.2 \mathrm{eV})$ created by TBs. To acquire a correct value of the mobility one should consider the direction of the TBs in the sample preparation. These findings can contribute to increase the reliability of mobility evaluation in $3 \mathrm{C}-\mathrm{SiC}$.

\section{Acknowledgments}

The work was supported by the Swedish Research Council (contract N ${ }^{\mathrm{o}} 1220100821$ ) and Swedish Energy Agency.

\section{References}

[1] Suemitsu M, Miyamoto Yu, Handa H, Konno A. J Surf Sci Nanotech 2009;7:311-13.

[2] Beaucarne G, Brown AS, Keevers M J, Corkish R, Green MA. Prog Photovolt Res Appl 2002;10:345-353.

[3] Podlasov SA, Sidyakin VG. Translated Izvestiya Vysshikh Uchebnykh Zavedenii, Fizika 1977;5:90-94.

[4] Lebedev AA, Abramov PL, Lebedev SP, Oganesyan GA, Tregubova AS, Shamshur DV. Phys B 2009;404:4758-60

[5] Moschetti G, Zhao H, Nilsson PÅ, Wang S, Kalabukhov A, Dambrine G, Bollaert S, Desplanque L, Wallart X Grahn J. Appl Phys Lett 2010;97:243510.

[6] Roccaforte F, Weng MH, Bongiorno C, Giannazzo F, Iucolano F, Raineri V. Appl Phys A 2010;100:197-202.

[7] Vasiliauskas R, Marinova M, Syväjärvi M, Liljedahl R, Zoulis G, Lorenzzi J, Ferro G, Juillaguet S, Camassel J, Polychroniadis EK, Yakimova R. J Cryst Growth 
$2011 ; 324: 7-14$

[8] Vasiliauskas R, Marinova M, Hens P, Wellmann PJ, Syväjärvi M, Yakimova R. Cryst Growth Des 2011;12:197-204.

[9] Jokubavicius V, Liljedahl R, Ou YY, Ou HY, Kamiyama S, Yakimova R, Syväjärvi M. Mat Sci Forum 2010;679-680:103-6.

[10] Furusho T, Sasaki M, Ohshima S, Nishino S. J Cryst Growth 2003;249:216-21.

[11] Blood P, Orton JW. The Electrical Characterization of Semiconductors: Majority Carriers and Electron States. London: Academic Press; 1992. 